\title{
Blockchain's Insurance Business Implementation
}

\author{
Akshay Sehgal \\ Executive, IT Department, IFFCO TOKIO General Insurance \\ Sector 29, Gurugram - 122001 \\ India
}

\begin{abstract}
This paper introduces the Blockchain technology and discuss its implementation for various Insurance business specific tasks. The emphasis has been laid to understand the process and transaction flow for the various business processes. Based on the integration of Blockchain technology with Business processes we can hope to leverage its features in order to generate digital solutions for the problems faced by the Insurance sector.
\end{abstract}

\section{Keywords}

Blockchain, Bitcoin, Insurance, Smart contracts, Shared Ledger

\section{INTRODUCTION}

Blockchain has been a broadly discussed technology, especially after its successful implementation in Bitcoin transactions. Although, Bitcoin holds apprehensions in the minds of the people but Blockchain as a technology has gained momentum because of its high innovation potential. Many startups and technology giants have started working to tap the unharnessed potential of Blockchain. Blockchain as a technology has been discussed largely for the banking area where its potential uses cases have been identified. However, it can also be used by insurers in order to spur growth, reduce administrative cost, enhance services, detect and prevent frauds. These are the major areas where innovative insurance products and services can be visualized. In this paper we would be analyzing the potential offerings of blockchain as a technology for the insurance sector laying emphasis on its usage in Claim Processing, Fraud Detection and KYCcompliance. Moving forward insurance industry could address some of the main challenges they are facing today - such as limited growth in mature markets and cost reduction pressures.

\section{WHAT IS BLOCKCHAIN?}

Blockchain is a distributed shared ledger that stores the transaction data received after consensus verification from all the nodes in the network. The approved and committed transactions are stored in a chain like structure with references to from the previous transaction to the new entry. This enables the transparency and replication of data in the distributed form. It was first used to resolve the problem of double-spend by combining peer-to-peer technology with public key cryptography. Since then it has evolved and is explored to revolutionize many fields like banking, finance, account management, etc.

To put it in other way, the Blockchain works on shared ledger technology which allow all the participants of the network to see the records stored in the digital ledger. It helped to resolve the problem of difficulty in monitoring asset ownership and transfer in a network. The solution provided by Blockchain is in the form of a consensus, replicated and shared ledger as shown below.

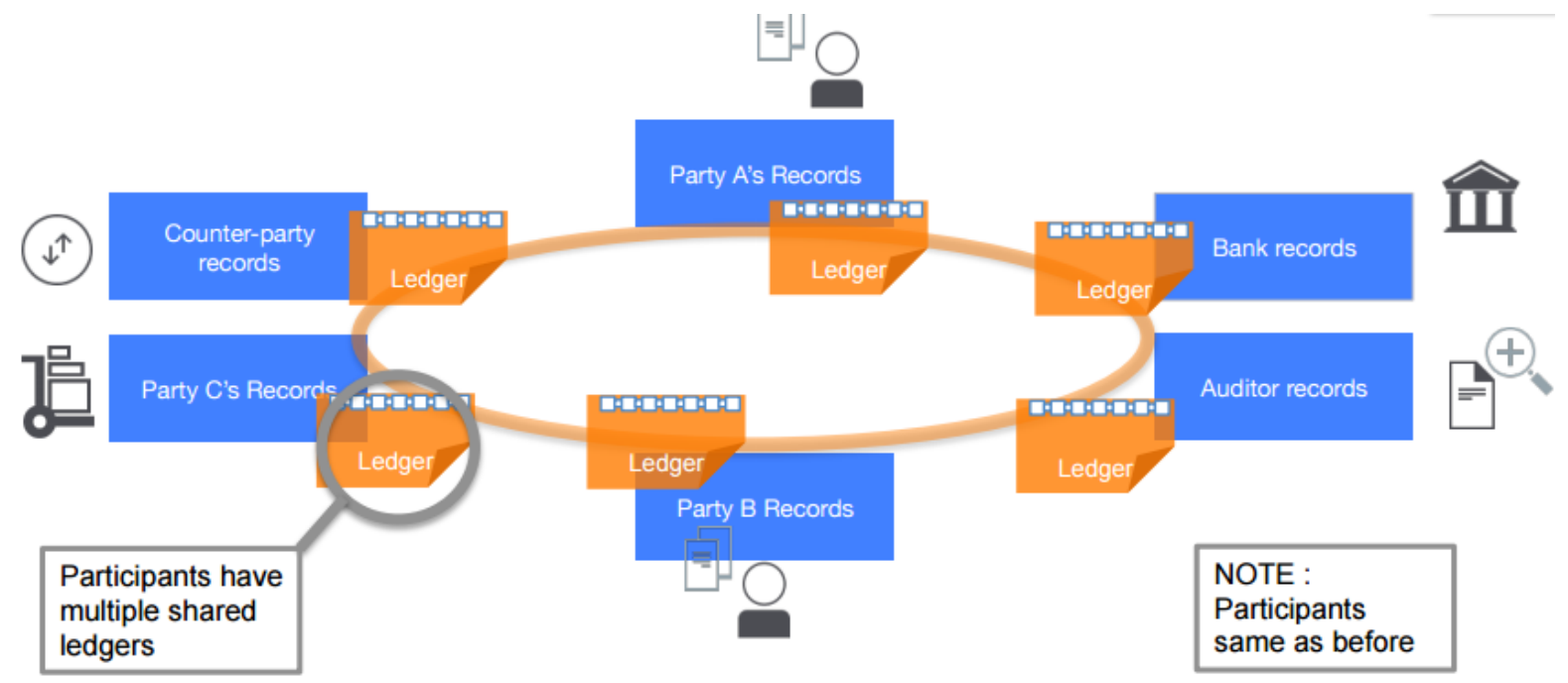

Figure 1 Shared Ledger in Blockchain 


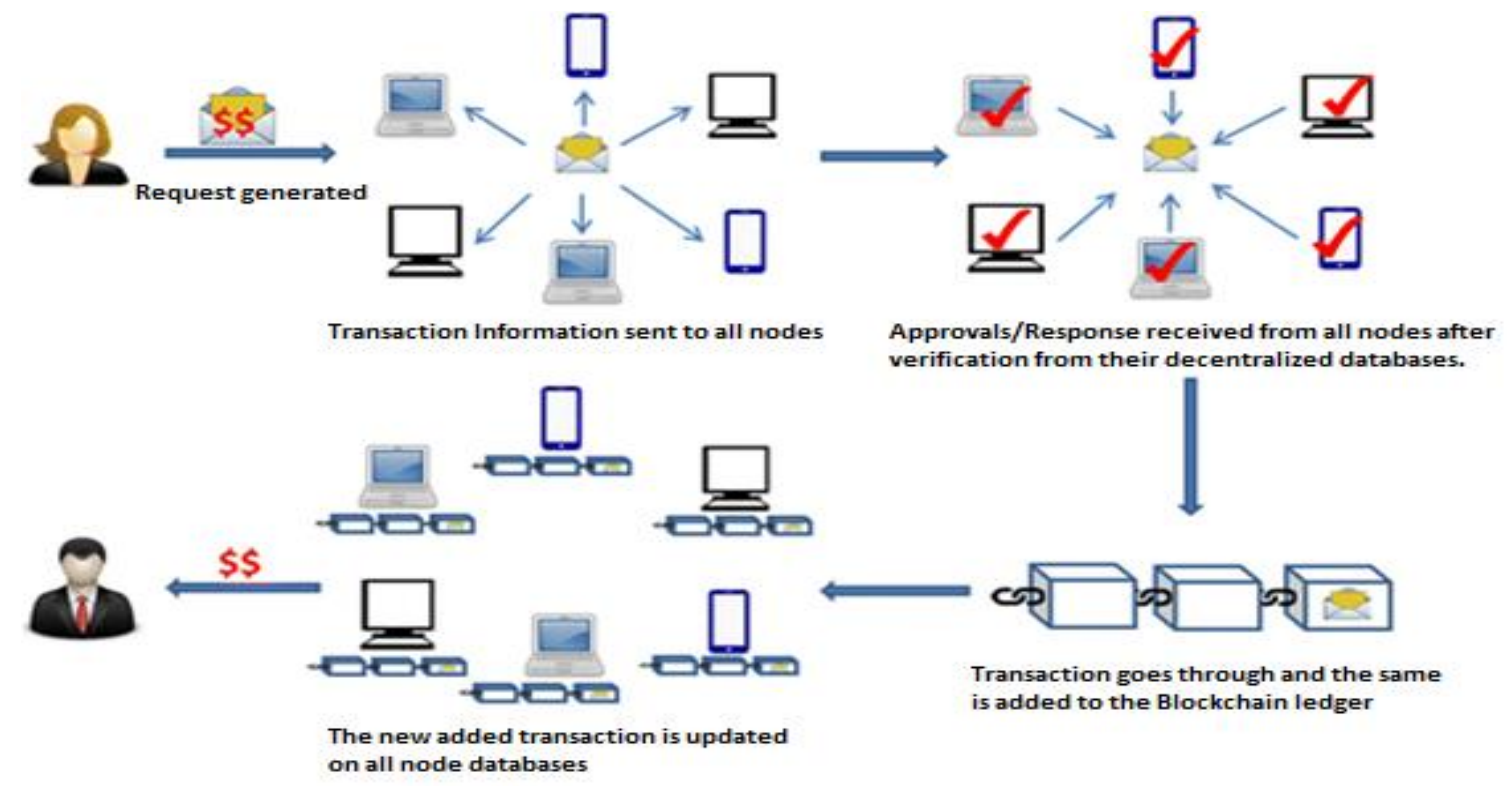

Figure 2 Consensus building from participating nodes before transaction

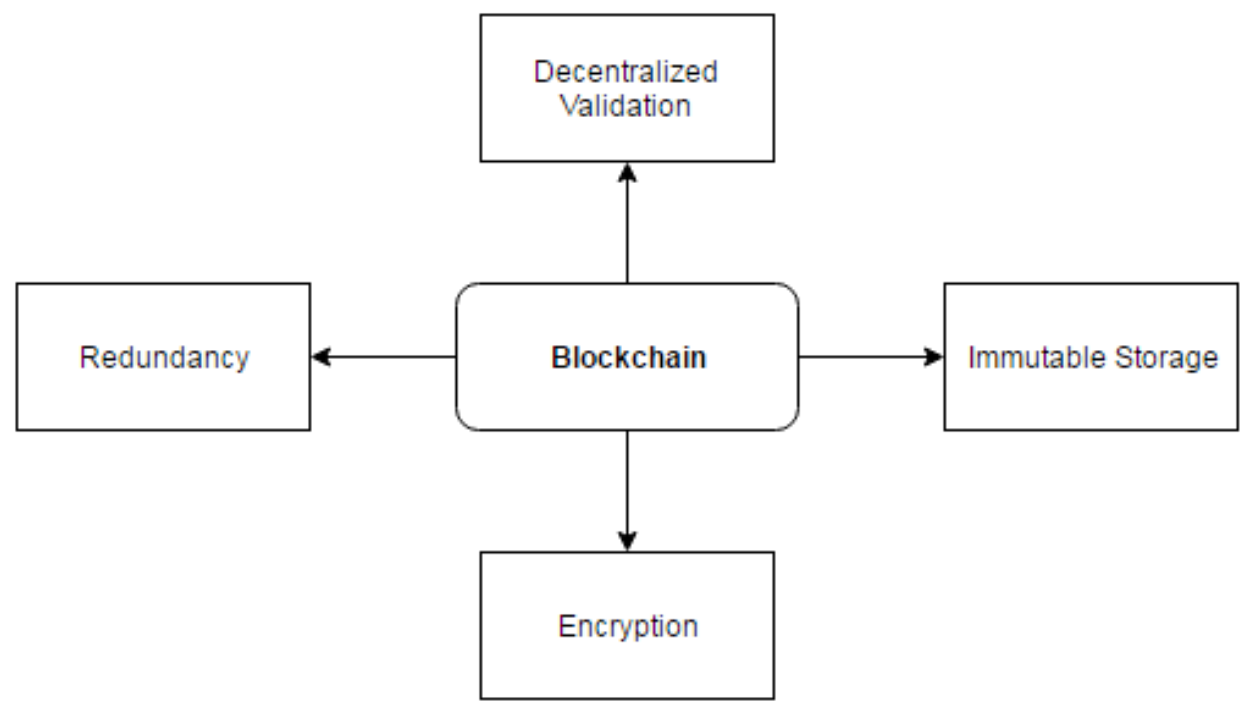

Figure 3 Characteristics of Blockchain

\subsection{Characteristics of Blockchain}

1. Decentralized Validation. Any transaction in the blockchain technology needs to be approved by the nodes in the network which can be other insurers, governmental organizations, financial institutions, or voluntary organizations. New transaction or data is packed into blocks only after consensual approval for all participating nodes.

2. Redundancy. The replication of data on each node or group of nodes helps prevent the situation of single point failure.
3. Immutable Storage. Each stored block in blockchain is attached to previous node and additional security is provided by generating a digital fingerprint to the new block from the previous block using a hash function which takes date and time as the input parameters. Hence any change in the block will require a change in the entire block chain and the replications of this data chain on node machines making it impossible for hackers to hack.

4. Encryption. Blockchain technology provides encryption of all the transaction related information among participating nodes and it is done in order to keep transaction data safe and make sure that it reach the intended recipient without being hampered. 


\subsection{How Blockchain can be used for business?}

At present, the insecurity faced by the customer is the apprehensions about misusage of data after handing it to the

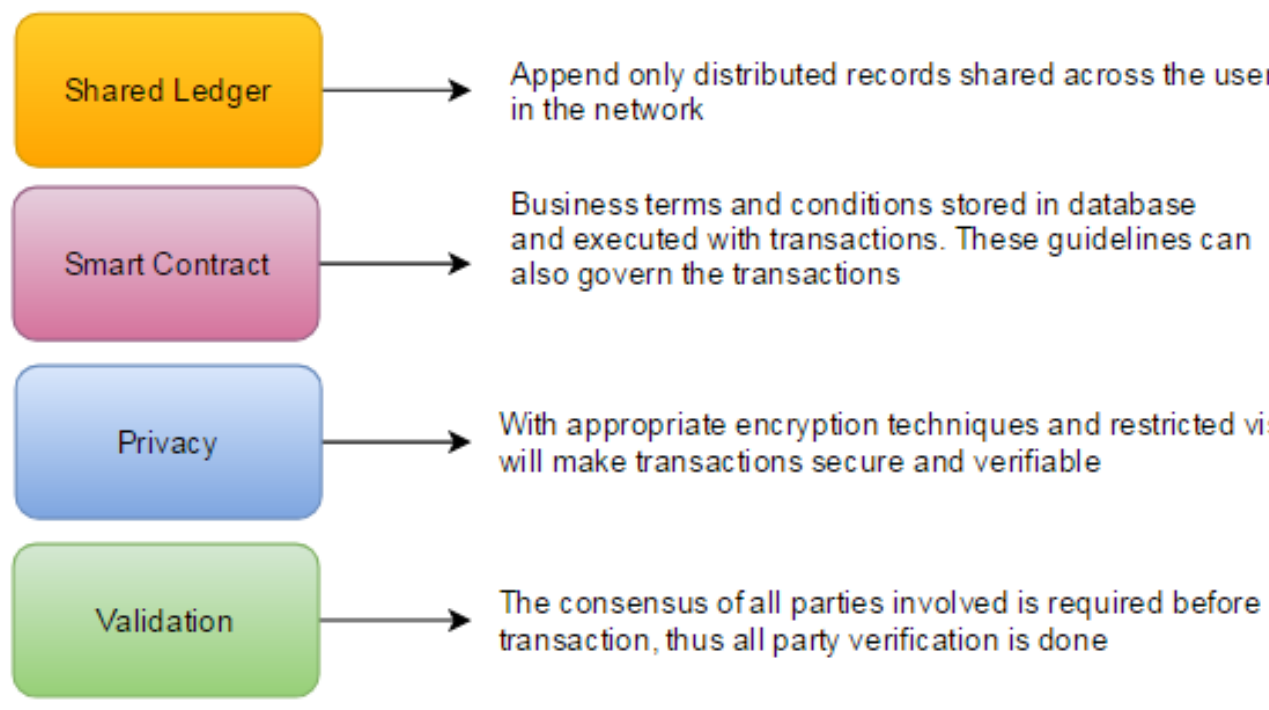

Figure 4 Features of Blockchain

The Blockchain provides the features of Shared Ledger, Smart Contract, Privacy and Validation to the business which can be used to automate the business along with the functionality of maintaining a shared ledger and readable records which can help in multiple areas. Here we will discuss how the Blockchain technology can be used in Insurance. Though there can be number of applications and the scope of further research can be to unearth more ways in which we can use this technology to make business more competitive, secure and digitalize the transactions. I have discussed three major applications or processes where we can use Blockchain technology and they are as follows.

\section{BLOCKCHAIN IMPLEMENTATION IN INSURANCE}

\subsection{Automated KYC Verification and Upholding Confidentiality of Data}

insurer or the need to re-enter data every time for verification. How this can be resolved? Blockchain can provide the solution to this by asking for the data from the customer which will be stored on his/her personal device only the instances of these values can be verified by the government authorities like UIDAI in India, Income Tax for PAN verification. This verification can also be extended by using the already stored data of the user in some other related transactions e.g. the financial records stored with banks, electricity department records, etc. Finally, the verification status can be shared with the insurer to move ahead with the KYC compliance. To scale and put this technology to its potential use, require sufficient number of parties to feed as well as reuse the verified data.

Another approach can be, once the KYC profile is verified, a customer can forward the verified data to other companies thus avoiding the need to verify data again and again, thus speeding up the process and increasing efficiency of on boarding of new customers. This way the confidentiality of

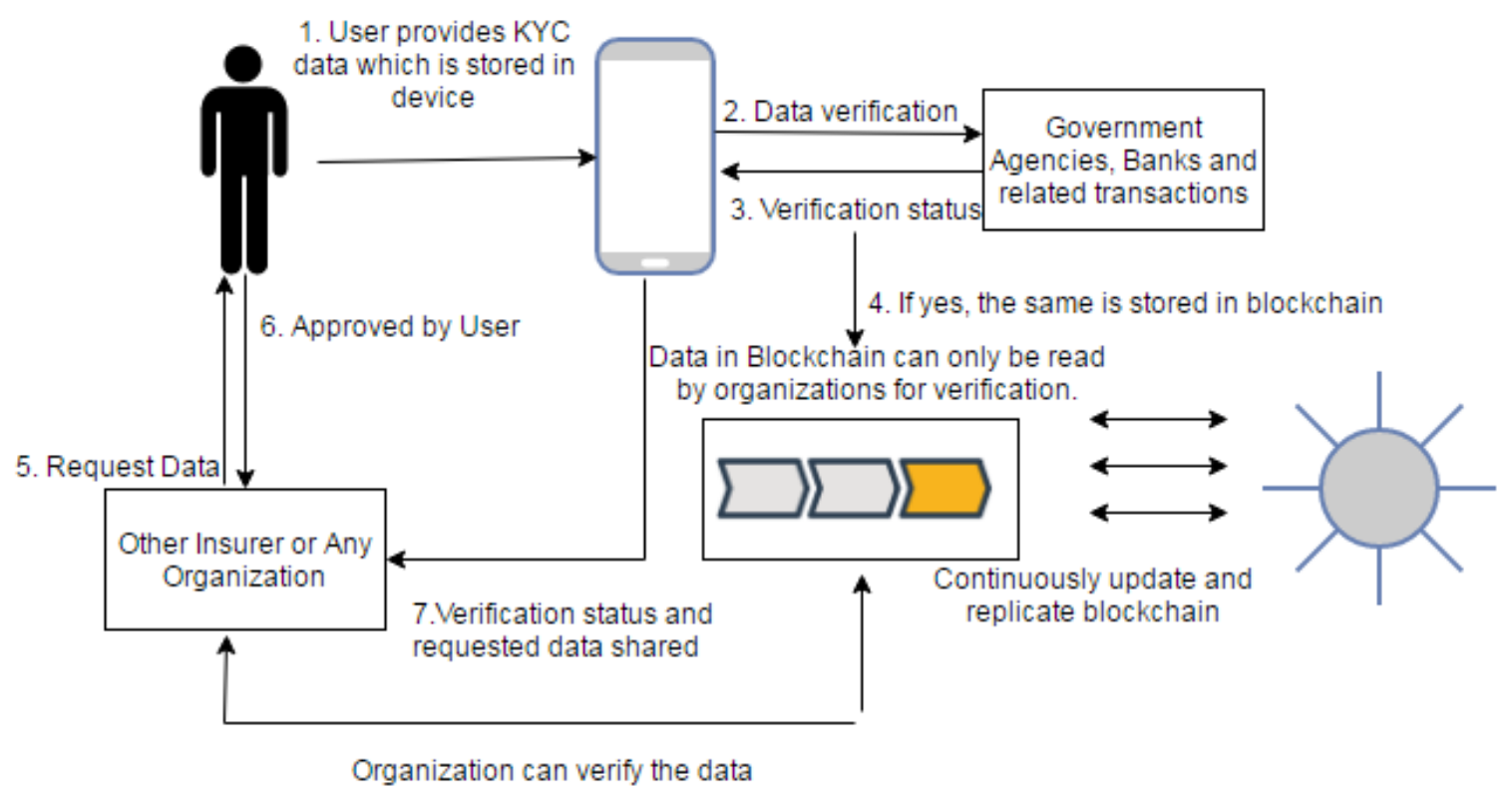

Figure 5 Process for KYC Compliance using Blockchain 
the customer is retained along with making it more transparent and verifiable at multiple fronts. The process described in Figure 5 emphasize on the process flow for KYC compliance and where Blockchain integrates in the process. The data and its verification status can be stored on Blockchain shared ledger and at the same time updated on individual machines for future verification. The process will be fully automated and ease the whole KYC procedure doing away the need to get documents verified every time.

\subsection{Claim Processing by using Smart Contracts}

the smart contracts. The AI and Analytics tools can interpret the past records and provide verified status for the claim to go through. Verification can also be done from various participating organizations and through authorized workshop. The relevant and required details of the claims can be shared with other insurers in the network to avoid multiple claim fraud and also to red flag insured initiating frequent claims. The automation in this business task will reduce cost substantially and Image detection and AI can also help to do away with surveyor reports and photographs as insured can himself/herself upload photos. Though this is not in the scope of this research and can be taken up separately.

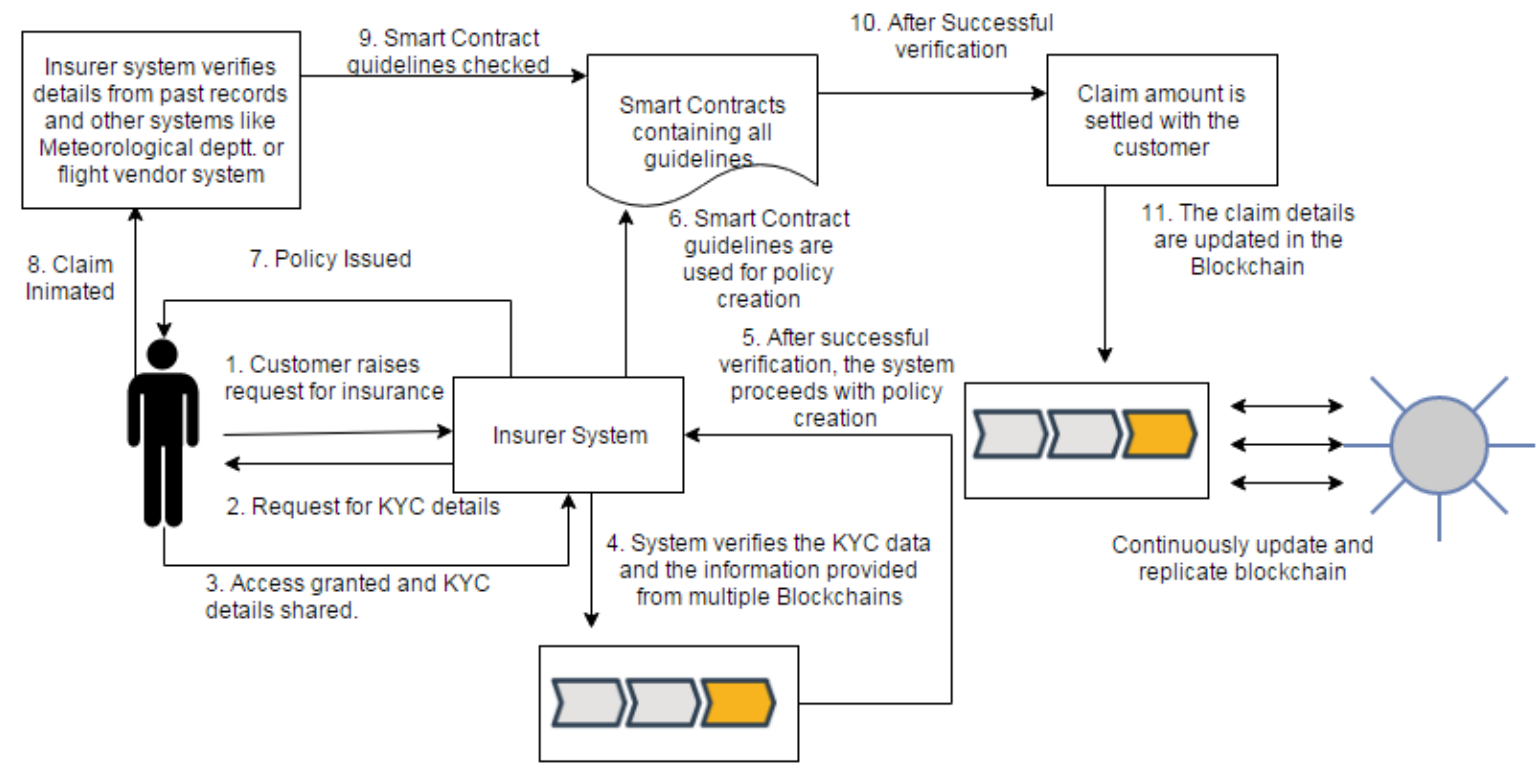

Figure 6 Process for Policy Creation and Claim Handling using Blockchain and Smart Contracts

Claim processing in Insurance industry is cumbersome and resource intensive task. Using Blockchain and AI, the industry can move ahead to automate the process of claim disbursal up to a certain amount. For claims like Travel Delay or Flight Cancellation in case of Travel Claim, the insurer can pick information from the systems of the flight service providers and initiate the claim for all the policies issued for the delayed/cancelled flights. This $\mathrm{P} 2 \mathrm{P}$ model of procuring the information and paying the claims is already in place using standard technology but with blockchain it will enhances the transparency and do away with the need of central authority that controls the operation. The startup Insure ETH demonstrated a P2P flight insurance policy built on blockchain with smart contracts.

In Emerging market, insurance policies with smart contracts and automated claim disbursal using blockchain can offer a substantial decrease in the cost of the insurance because of low handling costs. This can be done by following a set of rules in pre underwritten products and handling the claims after verification from the reliable data. For example, payouts of draught and floods can be made after verification from the weather/meteorological department database. Similarly, the insured could be offered claim payouts automatically when the car is repaired from the authorized workshop; this can be embedded in smart contract. This can energize the insurance sector by making transactions more transparent and cost efficient, thus offering benefits to the customers. The process has been described in Figure 6. The whole process gets automated and self-sustaining based on the rules defined in

\subsection{Fraud Detection}

According to this India forensic Research, the Insurance Sector in India loses 30401 crore of rupees every year due to frauds! In other words, every insurance company loses $8.5 \%$ of its revenues to the frauds. Similarly, Fraud accounts for 510 percent of claims costs for U.S. and Canadian insurers. Nearly one-third of insurers (32 percent) say fraud was as high as 20 percent of claims costs. According to the recent survey: 1 . There has been increased incidence of fraud over the last one year. 2. Fraud risk exposure is one of the major concern for the Insurance companies, the same has been highlighted by IRDA in India by emphasizing on the need to maintain the central repository of fraud cases. 3.Frauds are driving up the overall cost of the Insurers and that is why there is a whole lot of emphasis on use of digital technology for fraud prevention. Now, what is the role of Blockchain. Blockchain offers one among the many digital solutions available and can be used to maintain the central repository and in addition to it verify the data from all available nodes for its consistency. There are multiple ways in which blockchain can be implemented. One of the way is as follows. As per Blockchain principle the claim data or the claim lodged by the insured is stored in a decentralized database which is shared by all the Insurers, more the number of Insurers, more are the point of verification to check whether the claim with similar details is lodged multiple times or simultaneously with different insurers. Second, since all the claim data is stored in a Blockchain, each and every claim can be compared and tested with already filed claims and hence 
insured filing frequent or disproportionate claims can be detected. The system can also agencies, department of justice and other contributing and authorized agencies can update their data that is the information about fraudulent person, stolen goods, etc. in their blockchain. The insurer can access these blockchains for verification of proposal or claimant. This system when in place and fully functional can de-stress the Industry and will make the claim process more reliable and less fraud proof.
Blockchain can be utilized to solve the business problem and become worthy of its speculations. The technology is useful if only if it provides value and the same is explored by various startups working on similar ground. The scope of further research can be to expand the areas in which this technology can bring revolution and contribute to make business more resilient.

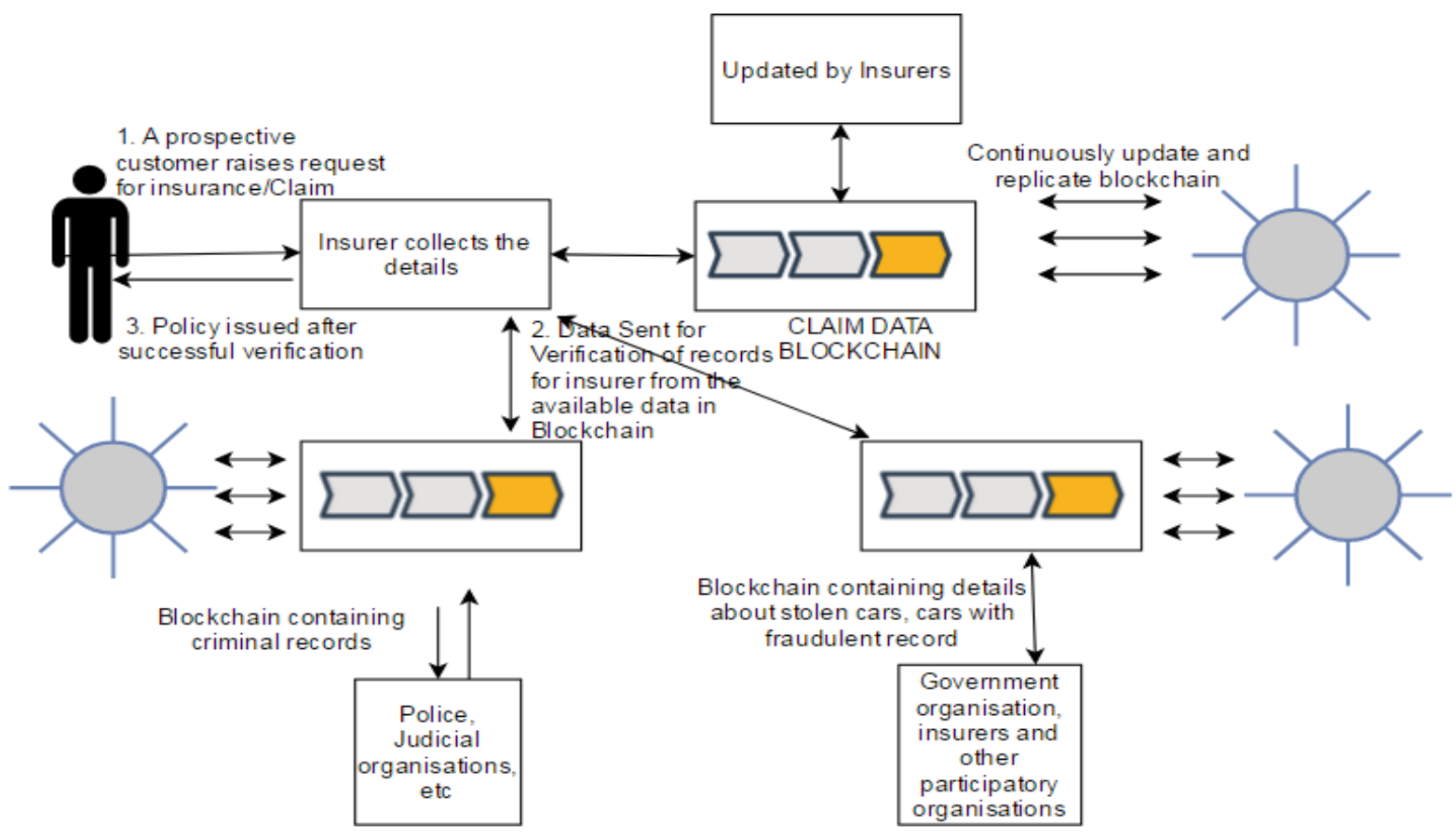

Figure 7 Process for Fraud Detection during Claim or Policy Issuance

\section{CONCLUSIONS AND RESULTS}

The aim of the paper is to introduce the Blockchain technology as a digital solution for the insurance industry, through this paper an effort has been made to discuss the implementation of the process for various Industry specific tasks. With the features like Decentralized validation and storage in the form of shared ledger, the data and information can be made to store and used from all the participating nodes making the transactions verifiable, transparent and automated at the same time maintaining the ledger of all transactions across industry. The smart contracts enable the features of policy issuance and claim handling digitally without human intervention based on certain rules enunciated in digital format. This would help enable the reduction in the amount of expenditure for the insurer, making them more competitive. The data stored at multiple fronts and the input from all the sources will make the industry more fraud prone and the same has been emphasized by various regulators by promoting the use of Business Analytics in order to reduce fraud. Blockchain adds more features to this by making data available from all sources like government agencies, past experiences, etc. and over the period it has the potential to be highly accurate for detecting fraud or an impostor. The technology offers multiple features and ways to enhance business on various fronts, what is required is the research to unearth the ways in which

\section{ACKNOWLEDGEMENTS}

Thanks to the Technical and Business Expert, Indraneel Neogi, Sr. Manager, ITGI for his insight on business processes.

\section{REFERENCES}

[1] Making Blockchain real for Business by IBMhttps://x9.org/wp-content/uploads/2016/02/BlockchainExplained-v2.09.pdf

[2] Statistical Report- http://indiaforensic.com/certifications/ india-loses-6-25-billion-to-insurance-frauds-anindiaforensic-research/

[3] Insurance Fraud data-http://www.insurancefraud.org/ statistics.htm

[4] Digital disruptions in Insurance - McKinsey

[5] Claims Wikipedia- https://en.wikipedia.org/wiki/ Insurance\#Claims

[6] What is Blockchain? - Introduction https://blockgeeks.com/guides/what-is-blockchaintechnology

[7] Introduction about Blockchain https://www.ibm.com/ blockchain/what-is-blockchain.html 
[8] Where is Current research on Blockchain Technology by Jesse Yli-Huumo, Deokyoon Ko, Sujin Choi , Sooyong Park, Kari Smolander- http://journals.plos.org/plosone/ article? id=10.1371/journal. pone. 0163477

[9] Chain Reaction - How Blockchain Technology might Transform - http://www.pwc.com/gx/en/financialservices/pdf/how-blockchain-tecnology-might-transforminsurance.pdf

[10] What does the future hold for blockchain and insurance? https://dailyfintech.com/2016/01/14/what-does-thefuture-hold-for-blockchain-and-insurance/
[11] The Present Use and Promise in Blockchain http://www.insurancejournal.com/news/national/2017/05 /16/451177.htm

[12] Blockchain - A Potential Game Changerhttps://www.cognizant.com/whitepapers/blockchain-apotential-game-changer-for-life-insurancecodex2484.pdf

[13] Are Blockchain Immune to malicious Attack- https://jfinswufe.springeropen.com/articles/10.1186/s40854-0160046-5 